\title{
Special issue on advance in multimedia and its application for convergence technologies
}

\author{
Seok-Hun Kim ${ }^{1}$ - Chung-Huang Yang ${ }^{2}$
}

Published online: 30 May 2015

(C) Springer Science+Business Media New York 2015

This special issue is intended to foster state-of-the-art research in the area of multimedia and its application for convergence Technologies (MACT). The MACT offers unprecedented opportunities for various modern multimedia applications and systems, such as ubiquitous computing technology, human-computer interaction, and convergence in multimedia technology, Bio-chips, RFID, and multimedia visualization. Its purpose is to solve the various problems of advanced MACT. This special issue focuses on advances in MACT.

This special issue has become one of the hottest topics in multimedia and application for convergence technologies. This special issue will also serve as a landmark source for multimedia and its application convergence and will provide a reader with the most important state-of-the-art technologies in areas of multimedia and application processing for convergence. We believe that this special issue will have high citation in the areas of multimedia and its application for convergence technologies.

This special issue includes 12 excellent papers from the selected papers of The International Conference on Computer Convergence Technology 2011 (ICCCT 2011) and from the regular submissions of other excellent researchers related to this special issue topic. The selected papers of ICCCT 2011 which were presented on October 20-22, 2011 in Seoul, Korea are revised and extended from the original version of conference papers.

Seok-Hun Kim

seokhun.kim1@gmail.com

1 Suwon Women's University, Suwon, Korea

2 National Kaohsiung Normal University, Kaohsiung, Taiwan
To contain a diverse collection of high-quality papers, all papers in this special issue are carefully reviewed through a rigorous peer-review process for publication.

We would like to express thanks to all people who have contributed their immense time and efforts in making this successful special issue. We thank all the authors who contributed their valuable papers for this special issue.

Finally, we would like to pay thanks to the Telecommunication Systems Journal Editor-in-Chief, Prof. Bezalel Gavish and staff for their encouragement and strong support during the preparation of this special issue. 